\title{
Brain Image Registration using Evolve Contourlet Transform: An Efficient Way to Register the Monomodal MRI-T2 Images
}

\author{
Abhinav Kumar ${ }^{1}$ and Dr D. Sasikala ${ }^{2}$ \\ ${ }^{1}$ PG Scholar, Indian Institute of Technology (BHU)/ ECE Department, Varanasi, India \\ Email: abhinavk.ece17@iitbhu.ac.in \\ ${ }^{2}$ Former Professor, CVR College of Engineering/CSE Department, Hyderabad, India \\ Email: anjansasikala@cvr.ac.in
}

\begin{abstract}
There are subsequently several image registration methods and they ensure excessive impact in the medical world. It is applied in the data examining in medicine, investigating in remote sensing, satellite imaging, etc., In this paper the algorithm is put forward registers' images of the identical modalities MRI T2 to MRI T2. There are some disadvantages of wavelet transform that have to be operated with the contourlet transform, but then that is now subsequently undertaking specific preprocessing after employing the contourlet transform harnessing the image enhancement technique using their low-subband that was got by the Laplacian pyramid as a part of decomposition and then reconstructing the image by using the reverse contourlet transform and then registering the reconstructed image with the targeted image by utilizing the geometrical transformations. The investigational outcome demonstrates that the Evolve Contourlet Transform imparts advantageous conclusions than the wavelet and also the traditional contourlet transform.
\end{abstract}

Index Terms: Registration, Wavelet Transform, Contourlet Transform, Evolve Contourlet Transform, Mutual Information, Correlation Coefficient

\section{INTRODUCTION}

The brain image registration is originating in the sector of medical image analysis in image processing. The research scope is very prominent in this unit as the more high-tech. Evolution in medical image analysis simpler remedy by the doctor. Medical science is professionally using the medical imaging in medicine and the major extents where it could toil out in terms of modalities would be on X-rays, Ultrasounds (US), Computed Tomography's (CT), Magnetic Resonance (MR) and Nuclear imaging as well. Subsequently, now in this exploration work, monomodal brain images are considered especially MRI T2 brain images are experimented. In so many areas, this will work as a key factor like in pharmaceutical industries, research labs in terms of medical imaging devices, research labs in terms of drug trials, research labs in terms of evaluating the quality of health care. [1]

Image registration is a process by which the pixels in two images just coincide to the matching points in the scene. So, by registration what it is inevitable is that if there are two imageries existing for example image $\mathrm{A}$ and image $\mathrm{B}$ or more images of identical entity in distinct time slot or selected from diverse devices placed at numerous spots. So, in these types of cases, if it is found that total unique image that will state more on the data contrast to additional image $[1,2]$.

Image registration is castoff for countless usages apart from that of the medical science, for illustration - in remote sensing, in satellite images, etc., In view of image registration, in a detailed manner in medical image analysis, for consideration of it clearly, a sample regarding a patient is procured. He goes for CT scan that day, the same day a patient goes for a CT scan after six months, but the location is altered, the CT scan center is reformed and that center after having a different CT machine with different resolutions, it is identified to all that the body is amending with time. For example, weight loss or weight gain. So, the CT scan must be unrelated with minor modification. Now the doctor requires in fact to relate amongst what occurred six months ago and what ensues that day for diverse points, like this registration is operated for orientation amid what comes about at what site before six months. At that flash moment the registration is sorted out point to point and predicts and displays that six months ago that point looked like that. The main criterion of registration is to blend the series of data with the discrepancies, if any or with their parallels into a solitary data. These collections of data are attained by appraising the equivalent scene or an entity apprehended at dissimilar point of time or from distinct viewpoints, in diverse coordinate systems. This research has been executed with two types of images; one is MR images and the other CT scan images.

Heaps of transforms or loads of techniques are readily available for performing the registration of images. Discussing the nature of the transformation, then there are chiefly two categories in general: Geometric transformations and Affine transformations. This is for the reason that aligning of images to intersect the universal aspects and add the variances if every, are to be highlighted for instantaneous perceptibility to the bare eye. This method can be grouped on four diverse facets similar to 1) the feature selection means extracting features from an image using their similarity measures and a correspondence basis, 2) the transformation function, 3) the optimization procedure, and 4) the model for processing by interpolation. So various algorithms are made known in distinctive research papers for image registration in which some are exceptional.

Image registration is categorized as intensity based and 
feature based image registration techniques. Further are categorized based on linear and nonlinear transformations.

The task accomplished in this artefact benefits contourlet transform in an evolved fashion for image registration. At this juncture roughly, pre-processing is accomplished formerly then the registration that alters the predictable procedure of contourlet transforms to accomplish image registration. Similarly, this article task stretches with the virtual investigation and relative analysis of wavelet transform and Evolve Contourlet Transform in medical image registration. The balance out of the paper or this work is systematically well-ordered as the following ensues. Section 2 provides an overview of the related works of image registration by deliberating some highlights of divergent techniques. Section 3 explicates the wavelet transforms and this elementary prevailing contourlet transform in image registration. Section 4 describes the steps forward for contourlet transform i.e., Evolve Contourlet Transform image registration. Section 5 exemplifies the investigational outcomes to verify the efficiency of this anticipated method in image registration and Section 6 accomplishes the article with a treatise.

\section{RELATED WORKS}

This research is executed to deliver an extensive investigation of the current resources available on Image registration methods [3].Consequently, there are countless techniques - to suggest a trivial number of image registrations using various transforms namely, Hough transform [4], Finite Fourier transform [5], Walsh and Fast Walsh Hadamard transform [6, 7, 8], Shift Invariant Feature Transform [9], Scale Invariant Feature Transform [10], Translation-Invariant Feature Transform [11, 12], Adaptive Polar and Modified Adaptive Polar transforms [8, 13], Wang Landau Adaptive Monte Carlo approach [14], Discrete Cosine transform [15], and thus various works were completed on Image Registration. This sector familiarizes approximately specific significant labels of research papers in image registration.

An appraisal of image registration techniques [ $1,16,17]$ and medical image registration techniques [1, 18, 19] are achieved. A computerized parallel image registration technique based on the Correlation of Wavelet facets [2] and Wavelet transforms [16, 18, 20, 21, 22, 23, 24]. Use of Multiresolution Wavelet Attribute Pyramids for Mechanized Registration of Multi-sensor Imagery [25]. Edge sensor assessment using pragmatic ROC curves [26], an association amongst edge detection and the nonlinear multiresolution depictions [27], Image coding using wavelet transform [24,28]. The nonsubsampled contourlet transform concept, blueprint, and usages [29]. Contourlets in Beyond Wavelets [30]. Image Registration by Contour Matching using Tangent Angle Histogram [31]. Medical image harmonizing - an analysis with categorization [32]. Approaches for registration, interpolation and interpretation of threedimensional medical image data for utility in 3-D display, 3-D modeling and therapy planning [33].

\section{Discrete WAVELET TRANSFORM AND INVERSE Discrete WAVELET TransForM}

As soon as a familiarization on digital communications ascends, for instance, an audio signal, the gorgeous smooth audio pattern is being renewed into an extremely sporadic stream of bits. The meaning here for sporadic is, when diffusing that flow of bits, on a communiqué network, in reality, initiating incoherence each time a bit alters over. So subsequently, for every single bit interval, there is a variation of waveform and hence, disjointedness at various levels, still if not in the function, in its derivative or in a second derivative whatsoever exist. the notion of signifying constant functions in terms of sporadic ones has its status in hands-on communiqué and thus, what Haar wavelet ensured is as follows. Owing to these origins of their virtuous aspects in [34], it can be emphasized that when associated with the traditional approaches, the Haar wavelet technique is extensively further sophisticated in concept, added appropriate in mathematical scheming and, furthermost of the whole lot, it is immeasurably expeditious in information handling .Equations (1), (2) and (3) are associated with Haar Wavelet Transforms.

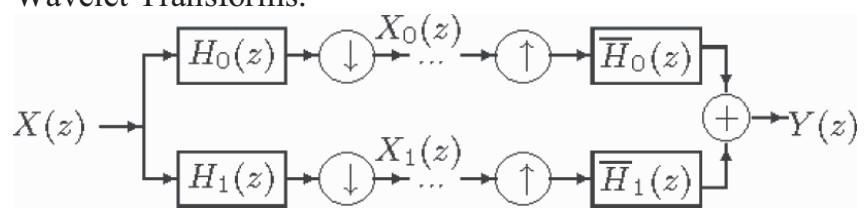

Figure 1. The low-pass and high-pass decomposition filters of Conjugate Quadrature Filter (CQF)bank of Discrete Wavelet Transform (DWT)

By means of Figure. 1 arithmetical lookout: Say $\mathrm{H}_{0}$ and $\mathrm{H}_{1}$ are the low-pass and high-pass categorization filters of Conjugate Quadrature Filter (CQF) bank of Discrete Wavelet Transform (DWT), correspondingly. $\mathrm{H}_{0}$ and $\mathrm{H}_{1}$ can be put up to make certain concerning the Prefect Reconstruction (PR) necessities, i.e.,

$Y(z)=X(z) z k$

where $X(\boldsymbol{z})$ and $Y(\boldsymbol{z})$ reveal the input and output signals, $k \in Z$. Yet, the aliasing in the output of disintegrated subband,$X_{0}(z)$ and $x_{1}(z)$, arise for the reason that of downsampling procedure that can be suggested by the subsequent investigations.

$$
X_{0}(z)=\left[X\left(z H_{0}(z)\right]+2=X_{0}^{\prime}(z)=\left[z^{-1} \mathrm{X}(z) H_{0}(z)\right]+2\right.
$$

where " $\downarrow 2$ " is a representation of the downsampling technique by the factor of " 2 ". If $X(z)$ is deferred by one sample, i.e., $\mathrm{X}^{\prime}(\mathrm{z})=\mathrm{z}^{-1} \mathrm{X}(\mathrm{z})$, the output $X^{\prime} 0(z)$ becomes

$$
\begin{aligned}
& X_{0}^{\prime}(z)=\left[z^{-1} X(z) H_{0}(z)\right]+2 \\
& =X\left(-z^{\frac{1}{2}}\right) H_{0}\left(z^{\frac{1}{2}}\right)+X\left(-z^{\frac{1}{2}}\right) H_{0}\left(-z^{\frac{1}{2}}\right) \\
& =z^{(-1 / 2)}\left[X\left(z^{(1 / 2)}\right) H_{0}\left(z^{(1 / 2)}\right)-X\left(-z^{(1 / 2)}\right) H_{0}\left(-z\left(^{(1 / 2)}\right)\right.\right. \\
& =z^{(-1 / 2)}\left[X_{0}(z)\right] \downarrow 2
\end{aligned}
$$

This transform embraces the filtering and down-sampling functions. As implied in Figure. 2, at each level of the wavelet categorization, four innovative images are fabricated from the exemplar image. The new-fangled images are named conferring to the filter (low-pass or highpass) that is put in to the real image in horizontal and vertical directions. For illustration, the LH image is an 
outcome of the gain of the low-pass filter in horizontal direction and highpass filter in vertical direction. Consequently, the four images shaped from each breakdown level are LL, LH, HL, and HH. The LL image is measured as a reduced version of the previous image as it holds utmost particulars. The LH image comprehends horizontal edge facets, still the HL comprises vertical edge facets. The $\mathrm{HH}$ encompasses high frequency data only and is stereotypically noisy and is, so, not beneficial for the registration [30]. In wavelet categorization only, the LL image is employed to yield the next level of breakdown.

\begin{tabular}{|c|c|c|c|}
\hline LL & HL3 & \multirow{2}{*}{ HL2 } & \multirow{2}{*}{ HL1 } \\
\cline { 1 - 1 } LH3 & HH3 & \\
\cline { 1 - 1 } LH2 & HH2 & \\
\hline & & \\
& LH1 & HH1 \\
& & \\
\hline
\end{tabular}

Figure 2. Image Compression Levels $-1^{\text {st }}$ level, $2^{\text {nd }}$ level and $3^{\text {rd }}$ level.

But then again, this system is unsuccessful in capturing the resourceful observable facts in imageries in the targets other than the horizontal and vertical directions. Do and Vetterli anticipated a proficient indicator multiresolution image exemplification termed the contourlet transform [30].

\section{Contourlet Transform}

The Contourlet Transform (CNT) offers a multiresolution and directional categorization; meanwhile it consents for a diverse quantity of targets at every calibration. First, a Laplacian pyramid is applied to gain control over the spot incoherence, formerly it is trailed by a Directional filter bank to associate spot incoherence into linear structures.

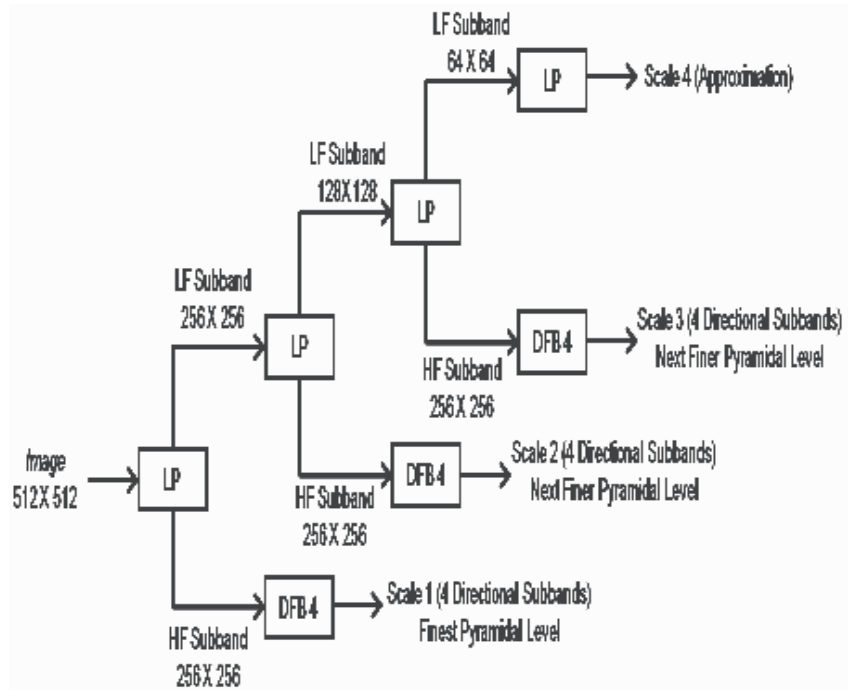

Figure 3. The flowchart of CNT for a $512 \times 512$ image
Owing to downsampling and upsampling, the contourlet transform is shift-variant, translation-invariant and scaleinvariant. Yet, shift-invariance is vital in image analysis functions such as edge detection, contour characterization, and image enhancement $[35,36]$. This aspect is just the reverse in the contourlet transform. Translation-invariant feature is essential for denoising $[11,12]$ and Scale-invariant feature is utilized for renormalizing signals [10]. These aspects are synchronized in the contourlet transform.

Contourlet transform is a resourceful indicator, targets multiresolution expansion that is digital friendly. Contourlet approximates multiscale, local and directional contour segments. The notion of contourlet transform emanated by means of the curvelet transforms that hinges on curvelet production that is the blend of numerous concepts [29, 37].

For achieving the contourlet categorization of an image, it is essentially obligated to have applied two transforms to it, the very first one is the Laplacian Pyramid (LP) and the second one is Directional Filter Bank (DFB) to yield orientation edge constituents of a segment in the interior of an image.

Contourlet Transform contributes a multiresolution, local and directional expansion of the image using Pyramidal Directional Filter Bank (PDFB). The PDFB fuses Laplacian Pyramid (LP) that depicts the spot incoherence, with a DFB that associates these incoherences into linear constructions.

Figure. 3, shows the flowchart of CNT for a 512 × 512 image. As shown in Figure 3, first stage of CNT is LP decomposition and DFB are in the second stage. LP scheme is shown in the following Figure 4. Here, the input image $x$ is the first lowpass filtered by analysis filter $H$ and then downsampled to give off a coarse approximation $a$. It is then interpolated and disseminated owing to the synthesis filter $G$. The resultant image is deducted from the previous existing image $x$ to get hold of the bandpass image $b$. This system can be recapitulated.

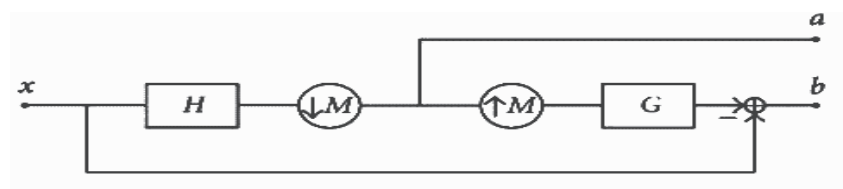

(a)

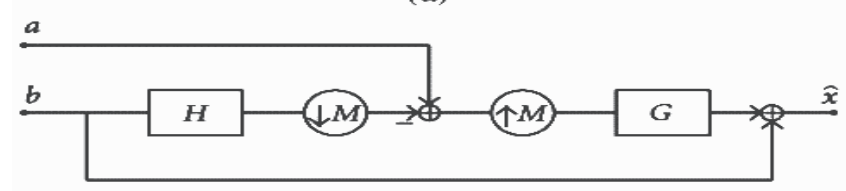

(b)

Figure 4. Laplacian pyramid. (a) One level of decomposition (b) The new reconstruction scheme

Equations (4), (5) (6) and (7) are associated with rigid transformation application. A rigid transformation applying on any vector $v$ engenders an altered vector $T(v)$ in term of

$$
T(v)=R v+t
$$

where $R^{T}=R^{-1}, R=$ orthogonal transformation and $t=$ vector forwarding the translation of the derivation.

An apt rigid transformation has the following determinant, 


$$
\operatorname{det}(\boldsymbol{F})=1
$$

i.e., the $R$ doesn't churn out a reflection, and stay alive in for a rotation. When an orthogonal transformation matrix begets a reflection, its determinant is $-\mathbb{1}[38]$.

\section{Anticipated Approach: Evolve Contourlet TRANSFORM}

The aim of image registration using Evolve Contourlet Transform is to assimilate complementary and redundant data from multiple images to generate a single image that encompasses of better-detailed data of the image than any of the specific source images. Here, both wavelets transform and Evolve Contourlet Transform registrations are implemented. Initially apply wavelet transform on an image and then perform reverse wavelet transform and by using this recreated image, registration is harnessed. In Evolve Contourlet Transform, first, apply the conventional contourlet transform, then rebuild the image by putting on the inverse contourlet transform. But, before the reformation, whatsoever image is acquired the lowpass and high pass sub-band or sub-images playing with that low subband and fundamentally, enhancing of the pixel values is resolved by applying fractional scalar multiplication. As the entire entity is a matrix by carrying out this the rebuilt image augment and impart more knowledge on the image. Obtaining this renovated image, image registration is then executed with the target image by using the same procedures of the above-mentioned geometrical transformations on the wavelet transform.

\section{A. Wavelet Transform for Monomodal Image Registration Process}

\section{Algorithm:}

Step 1:Put into operation wavelet transform on an image $I_{1}$ gaining an imageI $_{2}$

Step 2:Render reverse wavelet transform of $\mathrm{I}_{2}$ and by means of this $\mathrm{I}_{4}$ reconstructed image registration is utilized obtaining image $\mathrm{I}_{5}$.

Step 1 description:

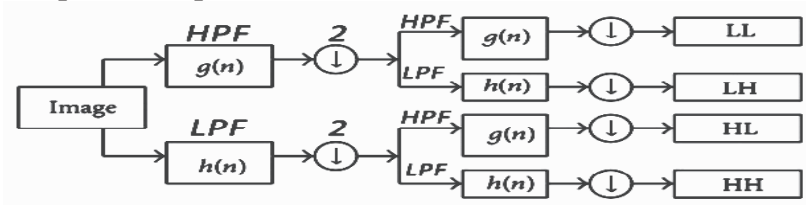

Figure 5. Discrete Wavelet Transform (DWT)

Figure 5. shows the approximation of DWT. The decimator down samples the input signal $g(n), h(n)$ by a factor of 2 .

Wavelet contexts of the Haar have remained illustrated to high-level measurement and ranking. The wavelet transforms have two types of coefficients, namely, 1. Scaling coefficients: created by manipulating the usual value of two neighboring models. They prove the approximation signal. 2. Wavelet coefficients: produced by scheming the contrast value of two neighboring examples. They explain the detailed signal. The Haar wavelet is a reasonable method and operating by the algorithmic calculation. Approximating the values of the scaling coefficients encompasses discovering the totality of two data models and distributing it by 2 . Correspondingly, assessing the values of the wavelet coefficients take in resulting in the variance of two data examples and sharing it by 2 .

Taking a discrete series that is signified as $f(x)$. This signal is a direct outcome of sampling of input data signal. In this subsequent discrete order, two in line models are engaged that are labeled as $\mathrm{X}$ and $\mathrm{Y}$. At that point, if the Haar transform is used on this order, the $\mathrm{X}$ and $\mathrm{Y}$, will be replaced by Average and difference values that are considered,

$$
a=(X+Y) / 2
$$

$$
\mathrm{d}=(\mathrm{Y}-\mathrm{X})
$$

Figure 6. indicates Haar Wavelet Transform. An order is chosen that is signified as $\lambda_{0 k}$, with number of example,

such that, $0 \leq \mathrm{k} \leq \mathrm{n}$.

The average and difference values are calculated for every pair, where $\mathrm{X}=\lambda_{02 k}$ and $\mathbf{Y}=\lambda_{02 k+1}$

As the n number of models is divided into odd and even models, the order will hold $\mathrm{n} / 2$, pairs. The difference and average values are specified as,

$$
\begin{aligned}
& \lambda_{-1, k}=\left(\lambda_{0,2 k}+\lambda_{0,2 k+1}\right) / 2 \\
& \gamma_{-1, k}=\lambda_{0,2 k+1}-\lambda_{02 k}
\end{aligned}
$$

Equation (8) and (12) does the average and Equation (9), (10) and (11) produces the difference of in line models.

The input data order $\lambda_{0} k$ is separated into two signal

elements $1 . \lambda_{-1, k}$ with $\mathrm{n} / 2$ averages and 2. $\gamma_{-1, k}$ with $\mathrm{n} / 2$

\section{differences.}

With the support of these average and difference values, the original signal can be improved. The average values are supposed to be the approximate signal depiction and the different values are measured as finer or complete signal depiction. In the occasion that original data order has predominant local consistency that indicates that the data discrepancy between the models is somewhat low. In such circumstances, the estimated signal depiction nearly looks a lot like the original data and the complete signal will be insignificant. Occasionally the complete signal will be near to 0 , in that occasion, it can be totally constricted.

The approximate signal order, $\lambda_{-1 k}$ is split more into 2

signal elements, $1 . \lambda_{-2 k}$ with $\mathrm{n} / 4$ averages and $2 . \gamma_{-2, k}$ with

$\mathrm{n} / 4$ differences and so on.

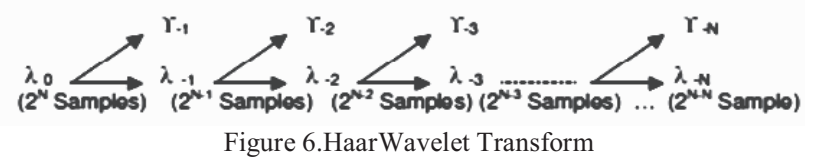


Save both ' $a$ ' and ' $\mathrm{X}$ ' and same way' $\mathrm{d}$ ' and ' $\mathrm{Y}$ ' in the matching place.

Originate by computing 'd'

Predict stage: $\mathrm{d}=(\mathrm{Y}-\mathrm{X}) \rightarrow \mathrm{Y}=\mathrm{d}$

(Substitute for the value of Y)

Here, the difference is considered for in line models, and the outcome will be kept in odd numbered places. So, revised as $Y=\mathrm{Y}-\mathrm{X}$.

Updated stage: $\mathrm{a}=X+\frac{d}{2} \rightarrow X=a$

(Substitute for the value of $\mathrm{X}$ )

So, revised as $X=X+\frac{d}{2} \rightarrow X=X+\frac{(Y-X)}{2}=(X+Y) / 2$

(Average value in line models)

Taking an example of a sequence of $\mathrm{n}$ sample, denoted as $\lambda_{0, \mathrm{k}}$, where, $0<\mathrm{k}<\mathrm{n}-1$. Considering the approximation level as 0 . At the next level that is 1 , this order can be transformed into a pair of other groups. It starts with dividing the input models to odd and even parts that is stated as lazy wavelet transform. Though, this does not aid the compressed signal depiction. In the next step, the prediction and updation methods of the lifting arrangement are originated that transforms the above order a) Approximation signal, denoted as, $\lambda_{-1, \mathrm{k}}$, where, $0<\mathrm{k}<\frac{\mathrm{m}}{\mathrm{z}}-1$. b) Detailed signal $\gamma_{-1, \mathrm{k}}$, where, $0<\mathrm{k}<\frac{\mathrm{m}}{\mathrm{m}}-1$.

Step 2 explanation:

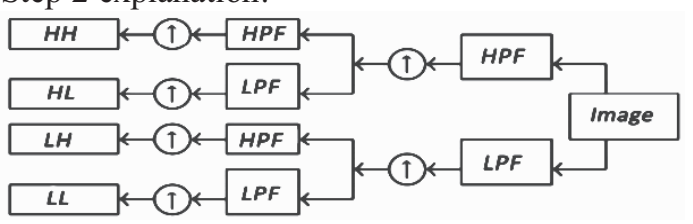

Figure 7.Inverse Discrete Wavelet Transform (IDWT)

The interpolator up samples the input signal $g(n), h(n)$ by a factor of 2 .

Also, the innovative values of $\mathrm{X}$ and $\mathrm{Y}$ can be attained by smearing inverse Haar transform,

$X=a-d / 2$
$Y=a+d / 2$
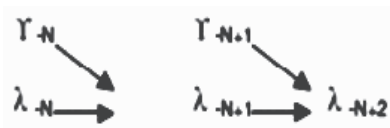

Figure 8.Haar Inverse Transform

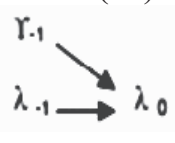

$X=X-\frac{Y}{2}=a-\frac{d}{2}=\frac{(X+Y)}{2}-\frac{(Y-X)}{2}$

(X substitute's average value)

$\mathrm{Y}=Y+X=d+X=(Y-X)+X$

(Y substitutes Difference value)

From Figure 7. Inverse wavelet transform is effortlessly realized from Equation (13) and (14) by merely swapping the sign in discrete wavelet transform. The Haar wavelet is wellthought-out as the modest orthogonal wavelet with minimalized provision in Figure 8. Thus, original values can be found with the aid of Equation (15) and (16).

The Inverse wavelet transformation is just performed in backward way of discrete wavelet transformation. With the support of lifting arrangement, it is no matter what but problematic to acquire inverse wavelet transform. It is straightforwardly realized by swapping the sign. Figure 7 and Figure 8 indicate the Haar Inverse Discrete Wavelet Transforms.

\section{B. Evolve Contourlet Transform for Monomodal Image Registration Process}

Algorithm:

Step 1: Put on the conventional contourlet transform on an imageI $_{1}$.

Step 2: $\mathrm{I}_{2}$ image is acquired the lowpass and high pass subband or sub-images playing with that low sub-band and fundamentally, enhancing of the pixel values is resolved by applying fractional scalar multiplication.

Step 3: Provide inverse contourlet transform of $\mathrm{I}_{3}$ and by means of this $\mathrm{I}_{4}$ renovated image registration is employed finding image $\mathrm{I}_{5}$.

In both the cases the Mutual information and correlation coefficient are measured, and the results are compared.

Step 1 clarification: Contourlet transforms Multiresolution and multidirectional expansion using nonseparable filter banks.

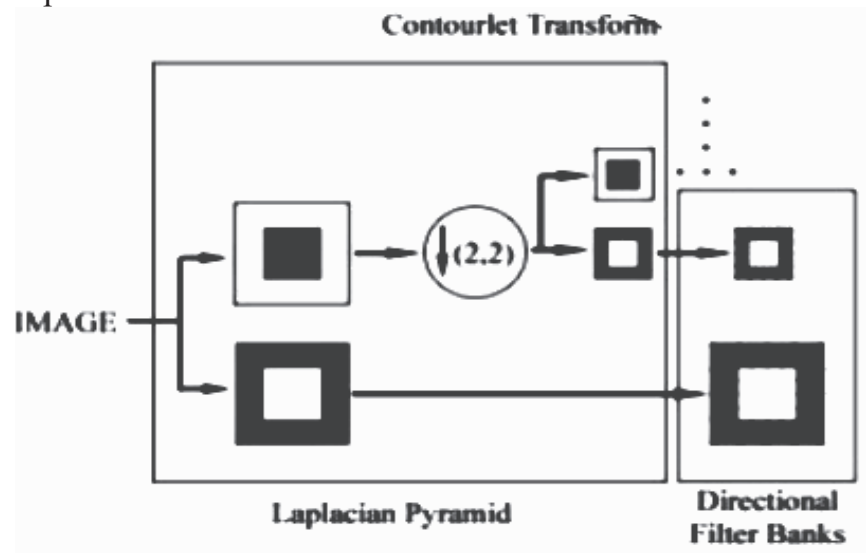

Figure 9. Contourlet Transform

From the Figure 9. Contourlet Transform the Laplacian Transform and the directional filter bank are expanded and shown in Figure 10 and Figure 11 i.e., the frequency partitioning representation of directional filter bank are shown below.

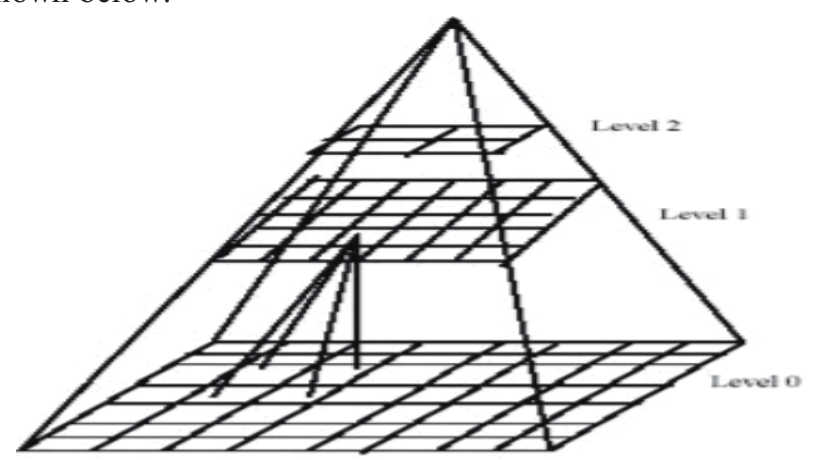

Figure 10. Laplacian Pyramid 


\section{Directional filter bank}

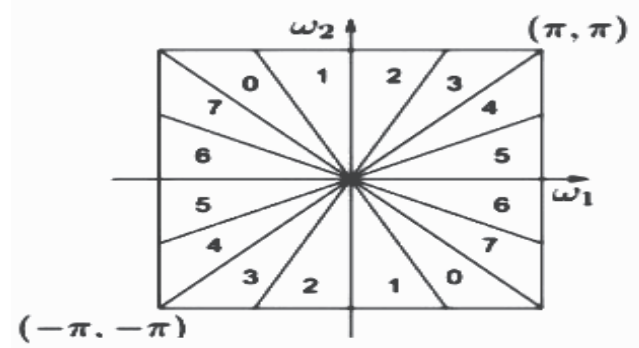

$$
S_{k}^{(l)}= \begin{cases}\operatorname{diag}\left(2^{l-1}, 2\right) & \text { for } 0 \leq k<2^{l-1}, \\ \operatorname{diag}\left(2,2^{l-1}\right) & \text { for } 2^{l-1} \leq k<2^{l},\end{cases}
$$

Figure 11. Frequency Partitioning

$$
Y_{i, j}^{\prime}=f\left(Y_{i, j}\right)
$$

Step 2: A threshold $\mathrm{T}_{\mathrm{i}, \mathrm{j}}$ is computed for each sub-band.

$$
f\left(y_{i, j}\right)= \begin{cases}W_{1 Y_{i, j}}(n 1, n 2) & \text { if } b_{i, j}(n 1, n 2)=0 \\ W_{2 Y_{i, j}}(n 1, n 2) & \text { if } b_{i, j}(n 1, n 2)=1\end{cases}
$$

Step 2 enhancement: Performs multi-scale salient features-based image enhancement by improving salient features like edges, lines, curves and contours using its anisotropy and directionality properties $[35,36]$.

Step 3 elucidation: The Inverse Contourlet Transform (ICT) is harnessed in Equation (18) in view of the improved directional sub-bands to acquire the ultimate improved image from Equation (17).

Denoting $\mathrm{C}$ and $\mathrm{C}^{-1}$ as the Contourlet and Inverse Contourlet transforms, correspondingly; $\mathrm{D}_{\mathrm{T}}$ is the diagonal matrix that, with threshold value $\mathrm{T}$, zeroes out irrelevant coefficients in the coefficient vector, whose total values are lesser than T. Let $\hat{X}$ the noisy high-resolution image; and $\hat{X}$ the denoised high-resolution image. The sparseness constraint by hard thresholding can be marked as Equation (19).

$$
\hat{X}=C^{-1} D_{T} C \hat{X}
$$

\section{INVESTIGATIONAL OUTCOMES}

Dissimilar medical image dataset used to achieve outcomes in this experiment. The dimensions of the dataset are changed. Consider the distinctive brain image acquired from the varied angle of the same patient at another time slots. The quality of the images attained after registration of images by this method has been tested using the Mutual Information and Correlation Coefficient.

\section{A. Mutual Information}

The Mutual Information (MI) between two variables deal with the volume of information that one variable comprises about another. High MI states a huge drop of ambiguity and low MI states an insignificant decrease in ambiguity and zero MI between two variables indicate that the variables are autonomous.

From Equation (20), it calculates the degree of dependence of the two images as MI, higher degree infers better quality, $I(X ; \boldsymbol{Y})=\Sigma_{y=Y} \Sigma_{x=X} p(x, y) \log \left(\frac{p\left(x_{y} y\right)}{p_{1}(x) p_{2}[y]}\right)$

- $X$ and $Y$ - Two discrete random variables

- $p(x, y)$ - Joint probability distribution function of $X$ and $Y$

- $p_{1}(x)$ and $p_{2}(y)$ - Marginal probability distribution function of $X$ and $Y$

\section{B. Correlation Coefficient}

There are two images, the first is template image and the second one is the candidate image, or say one is target image and another one is registered image. So, correlation states about the close relation between the pixels in the template and candidate image or target and registered image.

From Equation (21), Correlation Coefficient (CC) is a computation of how the forecasted data from a prediction model fit with the realistic data.

If there is no association between the predicted data and actual data, the $\mathrm{CC}$ is very low. As the association is increasing, the $\mathrm{CC}$ will also increase. The higher value of $\mathrm{CC}$ states concerning the better association between both the data.

$C(t, s ; \theta)=\frac{\sum_{x} \sum_{y}\left[I_{1}^{\text {new }}(x, y)-\overline{I_{1}^{\text {new }}}(x, y)\right]\left[\left[_{2}^{\text {new }}(x \cos \theta-y \sin \theta-t, x \sin \theta+y \cos \theta-s)-\overline{I_{2}^{n e w}}(x, y)\right]\right.}{\sqrt{\sum_{x} \sum_{y}\left[I_{1}^{\text {new }}(x, y)-\overline{I_{1}^{n e w}}(x, y)\right] \sum_{x} \sum_{y}\left[I_{2}^{\text {new }}(x \cos \theta-y \sin \theta-t, x \sin \theta+y \cos \theta-s)-\overline{\left.I_{2}^{n e w}(x, y)\right]^{2}}\right.}}$

$I_{1}^{M E W}(x, y), I_{2}^{n E W}(x, y)-$ Two new images that differ from each other by rotation and translation only.

$\hbar, s$-Shifting parameters between the two images.

$\theta$ - Rotation angle

$F_{1}^{\text {mEW }}(x, y), I_{2}^{\text {NeW }}(x, y)$ - Average structure value of the pixels in the overlapping parts of images $I_{1}^{\text {new }}(x, y), I_{2}^{n e w}(x, y)$

Tools- for the experimentations Matlab2017 R2017a were utilized.

For study Monomodal MRI T2 images are taken into account and the results are conferred and are instituted and are made known as below.

\section{Output I}

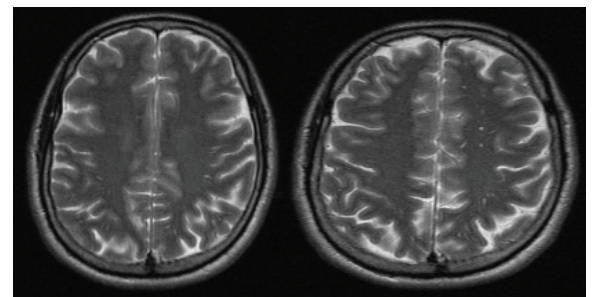

(a) MRI - T2 ImgT24.jpg

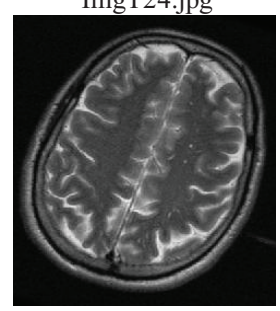

(d) Angle (theta) change in transformation $=18$ degrees

Figure 12. Registration using Wavelet Transforms for 18 degrees

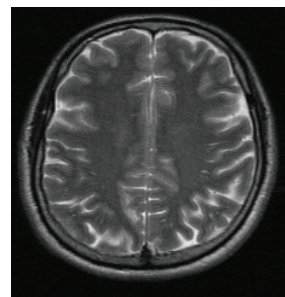

(c) Altered Image

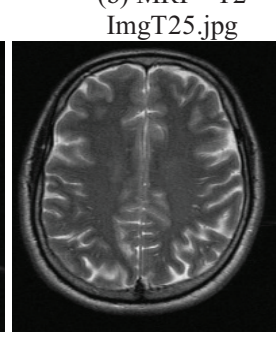

(e) Registered Image using Contourlet Transform 
From Figure 12. ImgT24.jpg and ImgT25.jpg, both after having the same modality MRI-T2 are registered using Wavelet Transforms with 18 degrees rotation to get the following output. Mutual Information $=1.4051$, Correlation Coefficient $=0.8640$ and Computation Time $=4.9780$

From Figure 13. the above-mentioned images are registered using Contourlet Transforms with18 degrees rotation to get the output given below. Mutual Information = 1.3680, Correlation Coefficient $=0.8646$ and Computation Time $=7.0196$.

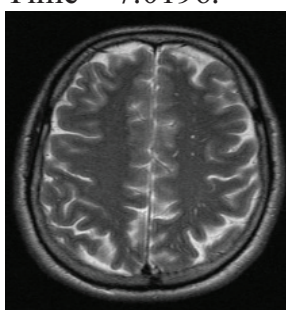

(a) MRI - T2 Img T24.jpg

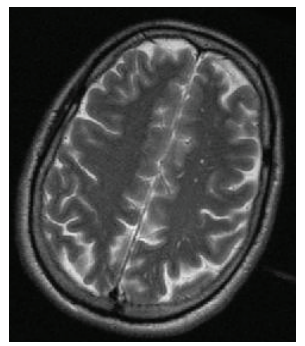

(d) Angle (theta) change in transformation $=18$ degrees

Figure 13. Registration using Contourlet Transforms for 18 degrees
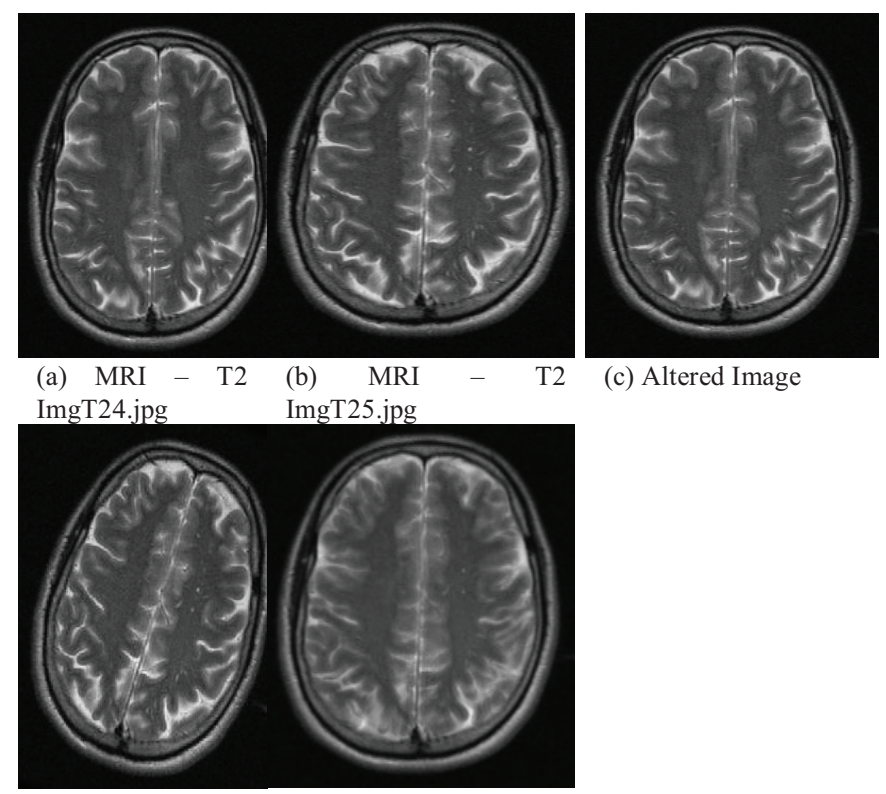

(c) Altered Image

(d) Angle (theta) using Contourlet transformation $=18 \quad$ Transform degrees

Figure 14. Registration using Evolve Contourlet Transforms for 18 degrees

From Figure 14. the aforesaid images are registered using Evolve Contourlet Transforms with18 degrees rotation to obtain the output given as: Mutual Information $=1.5283$, Correlation Coefficient $=0.8711$ and Computation Time $=$ 6.7361 .

From the Output I, though the computation time of the Evolve Contourlet Transform is a little high the MI and CC measures are high when compared to both registrations using Wavelet Transforms and Contourlet Transforms.

\section{Output II}

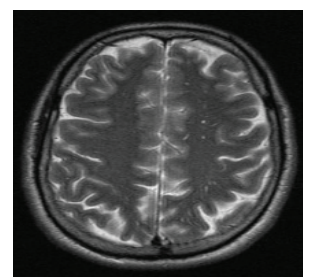

(a) MRI - T2 ImgT24.jpg

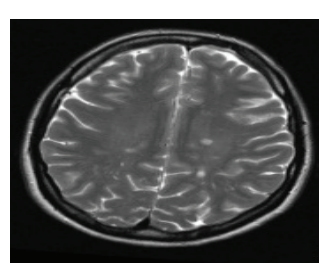

(d) Angle (theta) change in transformation $=7$ degrees

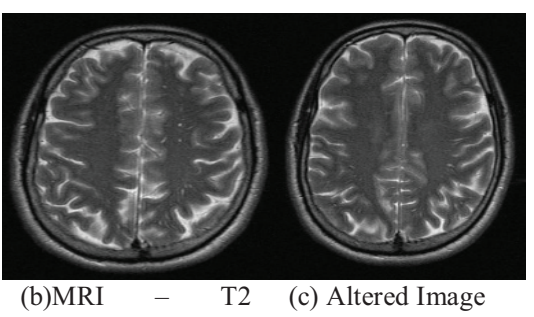
ImgT25.jpg

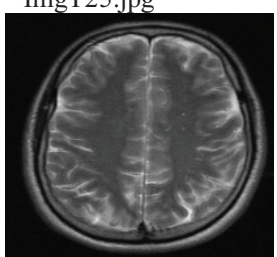

(e)Registered Image using Contourlet Transform

Figure 15. Registration using Wavelet Transforms for 7 degrees

From Figure 15. the aforementioned images are registered using Evolve Contourlet Transforms with 7 degrees rotation to obtain the output given as: Mutual Information $=1.0647$, Correlation Coefficient $=0.6365$ and Computation Time $=4.9513$
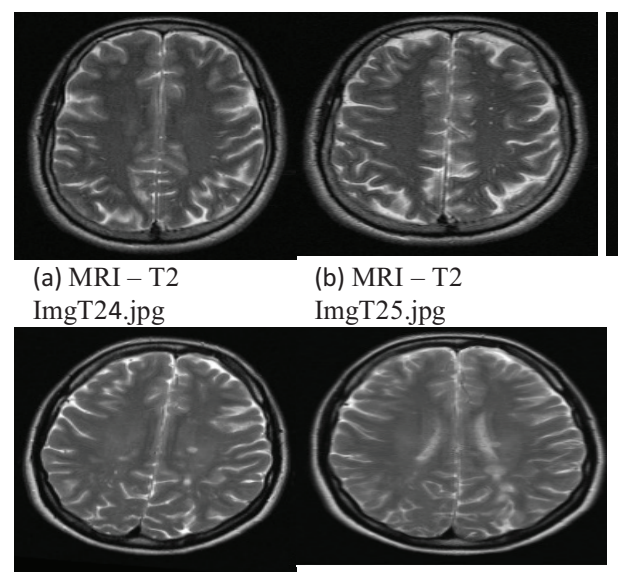

(d) Angle (theta) change in transformation $=7$ degrees

Figure 16. Registration using Contourlet Transforms for 7 degrees

From Figure 16. the said images are registered using Contourlet Transforms with 7 degrees rotation to obtain the output mentioned below as: Mutual Information $=1.0360$, Correlation Coefficient $=0.6379$ and Computation Time $=$ 5.9192 . 
From the Output II too despite the fact that the computation time of the Evolve Contourlet Transform is a little high but less than Contourlet Transform. The MI and CC measures are high too when compared to both the registrations using Wavelet Transforms and Contourlet Transforms.

Relating the consequences, it is evidenced that Evolve Contourlet Transform yields worthiest effects given away from amongst the three transforms.
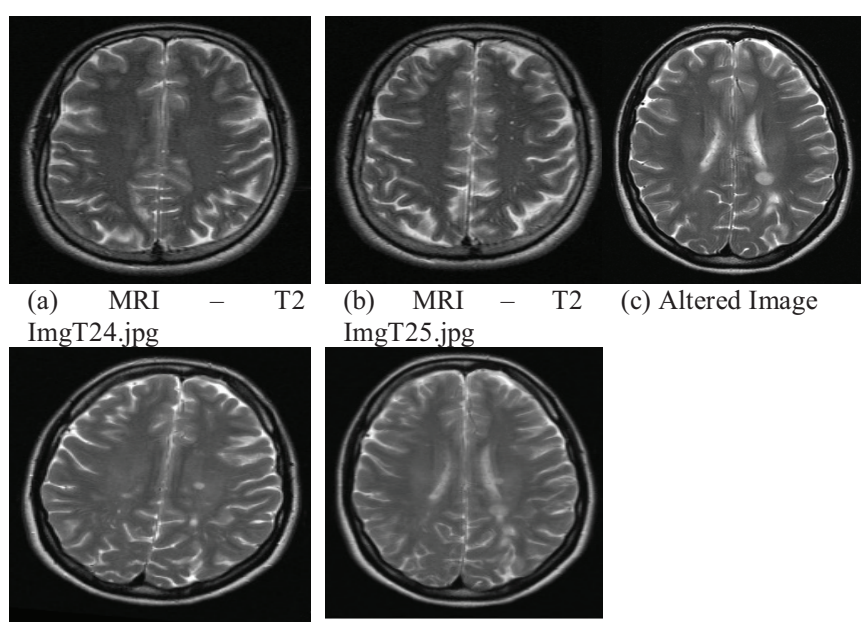

(d) Angle (theta) change in transformation $=7$ degrees

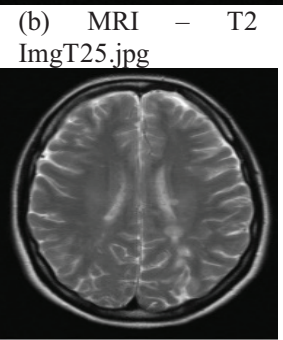

(e)Registered Image using Contourlet Transform

Figure 17. Registration using Evolve Contourlet Transforms for 7 degrees

From Figure 17. the aforementioned images are registered using Evolve Contourlet Transforms with 7 degrees rotation to obtain the output given below as : Mutual Information $=1.6480$, Correlation Coefficient $=$ $0.8462 \&$ Computation Time $=6.7157$.

\section{Future WORKS AND CONCLUSIONS}

Rigid image registration using the existing wavelet transform - the conventional contourlet transform is performed. Furthermore, in this paper, a new algorithm for medical image registration, an Evolve Contourlet Transform is proposed. After performing the rigid image registration using contourlet transform, Evolve Contourlet Transform too was performed using MRI T2 images. The quality of the images obtained by these techniques was analyzed and verified using the Mutual Information and Correlation Coefficient measures. The experimental results were illustrated by the imageries. It proves that the applied Evolve Contourlet Transform is a better approach than the conventional contourlet transform for medical image registration. The future work is focused on the improvement on the results by using some other transforms or by further changes in the Evolve Contourlet Transform Algorithm. Correspondingly testing can be done by adding a few more metrics along with the Mutual Information and Correlation Coefficient.

\section{REFERENCES}

[1] J. B. A. Maintz and M. A. Viergever, "A Survey of Medical Image Registration," Journal of Medical Image Analysis, vol. 2, no. 1, pp 1-36, 1998.

[2] J. L. Moigne, W. J. Campbell and R. F. Cromp, "An Automated Parallel Image Registration Technique Based on the Correlation of Wavelet Features," IEEE Transactions on Geoscience and Remote Sensing, vol. 40, no. 8, pp. 18491864, Aug 2002.

[3] Abhinav Kumar and Dr Anand Mohan, "Review of Image Registration Techniques", Journal of Emerging Technologies and Innovative Research (JETIR), vol. 6, no. 4, pp. 24-27, April 2019.

[4] F. Zana, J.C. Klein, "A multimodal registration algorithm of eye fundus images using vessels detection and Hough transform", IEEE Transactions on Medical Imaging, vol. 18, no.5, pp. 419-428, May 1999.

[5] E. De Castro, C. Morandi, "Registration of Translated and Rotated Images Using Finite Fourier Transforms", IEEE Transactions on Pattern Analysis and Machine Intelligence, vol. PAMI-9, no. 5, pp. 700-703, Sept 1987.

[6] Sasikala D, and Neelaveni R, "An Analysis of Brain Images using Fast Walsh Hadamard Transform", International Journal of Computer Applications (IJCA), 2011, Vol.13, No.1, pp 2329.

[7] Sasikala D, and Neelaveni R, "Correlation Coefficient for Registration of Monomodal Brain Images using Fast Walsh Hadamard Transform", Proceedings of IEEE International Conference on Communication, Control and Computing Technologies (ICCCCT 2010), Syed Ammal Engineering College, Ramanathapuram, Tamilnadu, India, $7^{\text {th }}-9^{\text {th }}$ October, 2010.

[8] Sasikala D, and Neelaveni R, "Performance Analysis of Brain Image Registration Technique using Fast Walsh Hadamard Transform and Modified Adaptive Polar Transform", Journal of Scientific and Industrial Research (JSIR), 2011, Vol 70, No.2, pp 123-128.

[9] Xiaolong Dai and S. Khorram, "A Feature-based Image Registration Algorithm using Improved Chain-code Representation Combined with Invariant Moments", IEEE Transactions on Geoscience and Remote Sensing, vol. 37, no. 5, pp. 2351-2362, Sept 1999.

[10] Sahil Suri, Peter Schwind, Peter Reinartz and Johannes Uhl, "Combining Mutual Information and Scale Invariant Feature Transform for Fast and Robust Multisensor SAR Image Registration",75th Annual American Society for Photogrammetry and Remote Sensing ASPRS Conference, March 9-13, Baltimore, Maryland, 2009.

[11] H. S. Stone, J. L. Moigne, and M. McGuire, "The Translation Sensitivity of Wavelet-based Registration", IEEE Transactions on Pattern Analysis and Machine Intelligence, vol. 21, pp. 1074-1081, Oct. 1999.

[12] A. Antoniadis, G. Oppenheim, "Translation-Invariant Denoising in Wavelets and Statistics", New York: SpringerVerlag, 1999.

[13] Sasikala D, and Neelaveni R, "Image Registration using Modified Adaptive Polar Transform", Procedia Computer Science, Elsevier, ScienceDirect, vol. 2, pp. 321-329, September 2010.

[14] Sasikala D, and Neelaveni R, "Brain Image Registration Techniques using Wang Landau Adaptive Monte Carlo Approach", Journal of Scientific and Industrial Research (JSIR), 2013, Vol 72, No.2, pp 114-121.

[15] Ruhina B. Karani and Tanuja K. Sarode, "Image Registration using Discrete Cosine Transform and Normalized Cross Correlation", Proceedings on International Conference and 
workshop on Emerging Trends in Technology (ICWET 2012), Thakur College of Engineering \& Technology, Mumbai, India, Feb 24-25, 2012, International Journal of Computers Applications, no.2, 2012.

[16] W. Pratt, Digital Image Processing, New York: Wiley, 2010.

[17] Zilnva B, Flesser J. "Image Registration Methods a Survey", Journal of Image and Vision Compute, vol.21, no.1 1, pp-977$1000,2003$.

[18] A. Aldroubi, M. Unser, "Wavelets in Medicine and Biology", CRC Press, Taylor and Francis Group, 1996.

[19] S. C. Strother, J. R. Anderson, X. Xu, J. Liow, D. C. Bonar, and D. A. Rottenberg, "Quantitative Comparisons of Image Registration Techniques Based on High Resolution MRI of the Brain. Journal of Computer Assisted Tomography, vol. 18, no. 6, pp 954-962, 1994.

[20] A. Cohen, C. Rabut, L. L. Schumaker, "Curvelets: A Surprisingly Effective Nonadaptive Representation for Objects with Edges in Curve and Surface Fitting", TN, Nashville: Vanderbilt Univ. Press, 1999.

[21] E. J. Candès, D. L. Donoho, " New Tight Frames of Curvelets and Optimal Representations of Objects with Piecewise C2 Singularities ", Communications on Pure and Applied Mathematics, pp. 219-266, Feb. 2004.

[22] I. Daubechies, "Ten Lectures on Wavelets, PA, Philadelphia: SIAM, 1992.

[23] Pavithra C and Dr. S. Bhargavi, "Fusion of Two Images Based on Wavelet Transform", International Journal of Innovative Research in Science, Engineering and Technology, vol. 2, no. 5, pp. 1814-1819, May 2013.

[24] M. Antonini, M. Barlaud, P. Mathieu, I. Daubechies, "Image Coding using Wavelet Transform", IEEE Transactions on Image Processing., vol. 1, pp. 205-220, Feb. 1992.

[25] I. Zavorin and J. L. Moigne, "Use of Multiresolution Wavelet Feature Pyramids for Automatic Registration of Multisensor Imagery, IEEE Transactions on Image Processing, vol. 14, no. 6, pp. 770-782, June 2005.

[26] K. Bowyer, C. Kranenburg, S. Dougherty, "Edge Detector Evaluation using Empirical ROC Curves", Journal of Computer Vision and Image Understanding, vol. 84, no. 1, pp. 77-103, 2001.

[27] A. Cohen, B. Matei, "Compact Representation of Images by Edge Adapted Multiscale Transforms", IEEE International Conference on Image Processing, Thessaloniki, Greece, 7-10 Oct 2001.

[28] B. N. Kingsbury, "Image Processing with Complex Wavelets", Philosophical Transactions of Royal Society London, pp. 2543-2560, Sep 1999.

[29] L. da. Cunha, Jianping Zhou and Minh N. Do, "The Nonsubsampled Contourlet Transform: Theory, Design, and Applications", IEEE Transactions on Image Processing, vol. 15, no. 10, pp. 3089-3101, 2006.

[30] M. N. Do and M. Vetterli, "Contourlets, in Beyond Wavelets", J. Stoeckler and G. V. Welland, (Eds), San Diego, CA: Academic Press, New York, 2003.

[31] Xiangyu Yu, Lihua Guo. "Image Registration by Contour Matching using Tangent Angle Histogram", IEEE Congress on Image and Signal Processing, Sanya, Hainan, China, 27-30 May 2008.

[32] P. A. Vanden Elsen, E. J. D. Pol, and M. A. Viergever, "Medical Image Matching- a Review with Classification". IEEE Engineering in medicine and biology, vol.12, no.1, pp 26-39, 1993.

[33] D. Vandermeulen. "Methods for Registration, Interpolation and Interpretation of Three-Dimensional Medical Image Data for Use in 3-D Display, 3-D Modelling and Therapy Planning", PhD thesis, University of Leuven, Belgium, 1991.
[34] ÜloLepik, and Helle Hein, "Application of the Haar wavelet method for solution the problems of mathematical calculus", Review article in Waves Wavelets Fractals Advanced Analysis, DE GRUYTER Open Access, vol. 1, pp. 1-16, January 2015.

[35] Zhi Qu, Yaqiong Xing and Yafei Song, "An Image Enhancement Method Based on Non-Subsampled Shearlet Transform and Directional Information Measurement", Information (Switzerland), vol. 9, 308, no. 12, pp. 1-15, December 2018

[36] E.P. Simoncelli, W.T. freeman, E.H. Adelson, and D.J. Heeger, "Shiftable Multiscale Transforms", IEEE Transactions on Information Theory, vol. 38, no.2, pp. 587607, March 1992.

[37] J. P. Zhou, et al., "Nonsubsampled Contourlet Transform: Construction and Application in Enhancement", IEEE International Conference on Image Processing, ICIP2005, Genoa, Italy, 1, 2005, pp. 469-472.

[38] O. Bottema\& B. Roth,."Theoretical Kinematics”. Dover Publications. Reface. ISBN 0-486-66346-9, 1990. 